preted in terms of familiar ideas; even to the most revolutionary of mankind, familiarity is a source of some satisfaction. The new theory is based on ideas utterly unfamiliar, and it might be urged that anything based on them must be the precise contrary to explanation. But if we ask why we are so ready to accept theories based on material analogy, we shall find our reason in the fact that such theories have actually turned out to possess the amazing property of predicting unsuspected laws. The theory of relativity also possesses that property. Ought we not to extend, so as to include it, our notions of the proper limits of physical theory, and to rid ourselves of the discomfort of unfamiliarity by the simple process of studying its ideas so closely that they become an integral part of our mental equipment?

It may be asked, Do theories, indeed, aim at nothing but satisfactoriness and prediction? Is not their object rather to discover the true nature of the real world? Such questions must be answered by questions. Do physicists (I say nothing of mathematicians or philosophers) believe that anything is real for any reason except that it is a conception of a true law or of a true theory? Have we any reason to assert that molecules are real except that the molecular theory is true--true in the sense of predicting rightly and interpreting its predictions in terms of acceptable ideas? What reason have we ever had for saying that thunder and lightning really happen at the same time, except that the conception of simultaneity which is such that this statement is true makes it possible to measure time-intervals? When these questions are answered it will be time to discuss whether relativity tells us anything about real time and real space.

\title{
The Relation between Geometry and Einstein's Theory of Gravitation.
}

\section{By Dorothy Wrinch and Dr. Harold JefFreys.}

$\mathrm{T}^{\mathrm{H}}$ HE term "geometry" has been used ever since the time of Euclid to denote two completely distinct subjects; but the formal similarity of their propositions has been so close as to obscure until recently the entire dissimilarity of their status in scientific knowledge. The Greek geometers seem to have been inspired originally by the need for a satisfactory method of surveying; at the same time, their logical turn of mind led them to present their results in the now familiar form of a deductive science. The characteristics of such a science are that a certain number of primitive propositions $p_{1}$, now called postulates, are stated at the beginning, and that from these, by a process of pure logic, further propositions $q_{1}$ are one by one developed. But this development is quite a separate process from that of deciding whether the primitive propositions are true or not, and if this is not done it is impossible to assert that the deduced propositions are true.

Different sets of primitive propositions $p_{2}, p_{3}, \ldots$. would give different sets of deduced propositions $q_{2}, q_{3}, \ldots$. and the complete working out of these is a science in itself; its results are all, therefore, of the form " $p_{1}$ implies $q_{1}$," " $p_{2}$ implies $q_{2}$," and so on. Euclid actually used in his development several postulates which he never explicitly stated, but which have been made explicit by modern writers; our present object, however, is not to indicate these, but to consider his geometry in the perfectly deductive form it would have had if he had actually stated them. We have noticed that in any other system in which any one of Euclid's postulates is false, many of his deduced propositions are also false. This, however, does not affect his method in the least; all his arguments are independent of the truth of the postulates, and in every case it is possible to assert-and this is all the modern geometer asserts-that if the postulates are true the propositions are true. A system like Euclid's is, therefore, a part of pure logic; the large division of pure logic that includes it as a very special case is pure geometry. Of the many systems of pure geometry now known, all are on just the same footing, and there is no sense in which any one of them is preferable to any other.

Euclid's contemporaries, however, were not interested merely in his logical method; they wished to identify the furrows in their fields with his lines, and the fields themselves with his surfaces; and to have some justification for this it was necessary to assume that his postulates were true of them. Only one example is needed to show how formidable an assumption this was. In order to prove one of his earliest propositions, Euclid assumes that a triangle can be picked up, transported bodily, and deposited on top of another. Imagine this process carried out when the triangles are fields! The impossibility of carrying it out implies that a most important proposition was not proved for the very case to which they contemplated applying his geometry, and hence that, so far as the knowledge of that day went, there was not the slightest reason for believing that geometry was applicable for its original purpose of earth-measurement. Yet its results, in so far as they were capable of being applied in actual surveying, seem to have been instantly accepted. Why? It may have been due partly to lack of disposition to criticise something that the critics felt they could not have done better themselves, a mental attitude that may perhaps still occasionally exist; but the chief reason was probably that some of the deduced propositions were directly verifiable, such as the proposition that the equality of corresponding No. 2677 , VOL. I06] 
sides of triangles in pairs implies that of corresponding angles; also many propositions about areas, expressed in numerical form, could be verified with some accuracy. As a result of these successes the whole system was accepted. But this could not have been a demonstration of the truth of the system; however many times a congruence proposition is verified for particular pairs of triangles, it will never be possible to prove it true for the next pair without the further assumption of some principle of empirical generalisation that is not included among the postulates.

Thus the grounds on which Euclid's results have been generally adopted in practice are not those of logical deduction from postulates known to be true $a$ priori, as was for centuries believed; they are largely based on empirical generalisation. Thus two sciences with different fundamental data, but with many formally similar propositions, have grown up: the original name of Euclidean geometry will here be retained for Euclid's own development, while the experimental science of measurement will be called mensuration. The latter was not developed on its own account, the reason being probably that it is a physical science, and that so long as a theory gives results in accordance with observation, physicists in general show little disposition to investigate the security of its foundations.

All experimental science depends on some postulate or postulates that imply that empirical generalisation is justifiable : no amount of experiment will enable us to make any inference unless we have some principle that enables us to generalise the results. Theories involving such a principle may be called extensive, while those not involving one may be called intensive. The latter include the whole of logic. Now no process of generalisation is used in pure geometry; every proposition is proved immediately and with complete certainty for every instance of its terms. Thus all pure geometry, Euclidean or otherwise, is intensive, while all physical sciences, including mensuration, are extensive. Thus, in the first great subdivision of scientific knowledge, geometry and mensuration fall on opposite sides, and nothing but confusion can arise from any attempt to treat them as identical. In geometry postulates are made about any point, or any line; in mensuration these are necessarily unverifiable, for they would have to be tested for every possible instance before they could be treated as postulates. They can thus be obtained only by generalisation from results that are obtained by experiment, and therefore cannot possibly be primitive propositions.

It may be remarked in passing that generalisation was condemned by traditional logic. The fact that scientific men have not studied its validity for themselves is the chief reason for the present chaotic condition of the theory of scientific knowledge. This important and basic principle appears to involve necessarily the notions of probability and combination of observations; yet in works purporting to be theories of scientific No. 2677 , VoL. Io6] knowledge these topics are habitually ignored altogether or else relegated to the last chapter. Much credit is due to Dr. N. R. Campbell for placing the theory of probability in its proper position in his recent "Elements of Physics." The value of his treatment is injured by the adoption of the Venn definition of probability, which is logically unsound and scientifically inapplicable; but to accord probability its true status in scientific knowledge is the first great advance in the theory.

This neglect of mensuration, while geometry was making rapid progress, has led physicists to give undue attention to the latter subject and an undue physical status to its concepts, especially to that of space. Metrical geometry has come to be regarded as the theory of the measurement of space; but space has no status among the subjectmatter of mensuration. A physical measurement of length is always of the form: "When the zero mark on the (so-called rigid and straight) scale is in contact with the particle $A$, and the edge of the scale is in contact with the particle B, B lies between the $n$th and $(n+\mathrm{I})$ th divisions of the scale." This involves no reference to space. Even the notion of rigidity, which is often regarded as spatial, is not so in practice. It is an experimental fact that many bodies exist which under ordinary treatment have the property that, if the distance between two points of the one can be made to include the distance between two points of the other, in some configuration, then, however the bodies are displaced, this remains true; and our rigid scales are such bodies. This, with the purely mechanical process of scale dividing, is all that is needed to make measurement possible. In actual physical calculation, again, we simply use the measures themselves without any reference to "space" ; and the final result can be stated wholly in terms of such measures. If the notion of space is introduced at any stage of the investigation, it necessarily eliminates itself before the close. There are, of course, many so-called measurements of length in physics, such as the diameter of the earth and the distance of the sun, which cannot be made by means of rigid scales; these are not, in the strict sense, measured at all, but inferred from measures of certain angles by means of physical laws obtained from other experiments. Time, as actually used in physics, is on a similar footing to distance measures, and not to space.

The relations between the measured positions of bodies at different times form the subject of dynamics, which reduces to mensuration in a special case. It is an extensive science, and therefore epistemologically quite different from any four-dimensional geometry, which must from its nature be intensive. For this reason we consider that the theory of gravitation must be treated on extensive lines, and disagree with Prof. Eddington's presentation of Einstein's theory as a section of geometry. The principle of the irrelevance of the mesh-system, in particular, does not seem to be presented in its true light in his 
" Space, Time, and Gravitation." This principle states that there are relations between the coefficients in the formula for $d s^{2}$ which hold, no matter what system of co-ordinates is chosen. Eddington regards the co-ordinate system as decided upon arbitrarily, having no real physical importance, and always eliminating itself in any actual physical process, while the only thing that has physical importance is space-time, which he treats on purely geometrical lines. From this point of view the principle is evidently correct; but it is not the point of view of physics. In physics the co-ordinate systems actually chosen are adopted entirely because they give specially simple forms to relations between measured quantities, and thus are not chosen arbitrarily. It would therefore be conceivable, and indeed not improbable, that there could be no relations between the $g$ 's that would not have special forms with ordinary coordinate systems. On the other hand, the properties of space-time never appear in physical laws; thus it is space-time that eliminates itself when the problems are reduced to terms of measurement, and the irrelevance of the meshsystem is a proposition, not about the unimportance of convention, but about physical measurements themselves. Hence it is a part, not of geometry, but of dynamics, and can be arrived at only by extension. Now that the predictions of the theory have been verified, the proper course to adopt is to derive the form of $d s^{2}$ from the experimental results that these express, and to regard the principle as an interesting experimental result.

Einstein's own presentation differs somewhat from Eddington's, but is also open to some objections. In his "Relativity, the Special and the General Theory" (English translation) Einstein states on p. 2 that he uses the term "geometry" in its usual sense of the logical connection of ideas (? propositions) among themselves. Then, on p. 3 , he introduces the further proposition that two points on a practically rigid body always correspond to the same distance, however the body may be displaced, and considers that this converts geometry into a branch of physics. This is not the case; it can only give series of implications between propositions of the truth of which we are ignorant, until some of these propositions have been proved by sensory experience, which is not a part of geometry; they also require a process of generalisation to yield results of sufficient generality to form the basis of a geometry, so that a science reached in this way must be extensive.

On p. 9 of his book, Einstein's attitude towards "space" is closer to ours than to Eddington's, for he resolves to shun the word entirely, admitting that he cannot form the slightest conception of its meaning, and replaces it by the notion of position (i.e. measured position) relative to a practically rigid bodv of reference. In the latter part of the book he appears to regard space, not as a primary entity of Nature, but merely as a conventional construct. composed of the aggregate of all possible values of the three position co-ordinates. In this form the notion may be useful in theoretical work, but we cannot attribute any ultimate physical importance to a thing we have constructed ourselves. In fact, he makes it clear on p. 60 that the principle of relativity is a postulate to be tested by experience: a suggestion offered as possibly true, but with no a priori necessity about it. Its validity, in the opinion of its author, rests wholly on the success of its physical predictions. Consequently, as was stated above, the correct procedure now is to deduce the theory from the facts originally predicted by it, with whatever further postulates may be necessary.

Although we have criticised the chief current expositions of the Einstein theory, it is the omission of physicists to provide any satisfactory analysis of the foundations of their own subject that is chiefly at fault, and the remedy is a proper discussion of the relation of physical laws to the observations on which they are founded, and of the probability of inferences based upon them.

We may devote some attention to the question of the comparison of standards at different places, which must play an important part in any theory of measurement. Before the publication of the special theory of relativity, the accepted view was comparatively simple: the measured length of the conventional rigid bar was supposed to be the same however it was displaced and turned, and the same applied to the period of vibration of a clock or an atom (in the former case subject to the known influence of temperature and gravity). This gave a convenient basis for comparison of standards, for all could be expressed in terms of some standard instruments. The special theory, however, showed that this statement is not merely inconvenient as a working rule, but also demonstrably false, for a bar must have different measured lengths according as it is moving relative to the observer along or across the direction of its length. The possibility of a comparison was restored by Einstein's system of light signals; time and length standards were supposed unaltered by displacement, provided the observer was moving with them. But whether he was moving with them or not, a certain interval $f d s$, measured between consecutive vibrations of the clock or between points on the bar, remained unaltered. Also the condition that $j d s$ was stationary for small variations in the path was found to give the conditions satisfied by a particle moving at a great distance from matter.

Thus this differential element $d s$ had a dual importance in the special relativity theory. In the general theory this is generalised, but it may be noticed that it is just possible that the two rôles may really be separated in a gravitational field. The consequences of this would be peculiar. It is well known that the assumption that $d s$, taken through a period of vibration of an atom, is independent of position, leads to the prediction of a shift of solar spectral lines; though this is so mixed up with shifts arising from other causes NO. 2677 , VOL. IO6] 
that it is impossible to say definitely whether it has been observed or not. But it is not so well known that the assumption that would lead to no spectral shift leads to the result that the wavelength on emission of light from a particular type of atom is a function of position; thus the aban- donment of $d s$ as the fundamental measured quantity would not make it possible to carry both length and time standards about unaltered. Probably the difficulties arising from the hypothesis that $d s$ does not play two parts are so great as to render it quite unplausible.

\section{The Metaphysical Aspects of Relativity.}

\section{By Prof. H. Wildon Carr.}

$\mathrm{T}^{\mathrm{H}}$ HERE is a possible misconception in the application of the term "metaphysical" to the new principle of relativity which it is advisable to clear up. In the great era of the triumphant advance of the positive sciences, which began about the end of the first third of the nineteenth century, metaphysics was decried as the main obstacle to scientific progress. Following the lead of Auguste Comte, the workers in the sciences held it up to scorn as obscurantism. The derision and reproach which were then poured on it have clung to it ever since. There are many to-day who acknowledge, indeed, that metaphysics must be assigned a place in the hierarchy of the sciences, but interpret the Aristotelian definition, "that which follows or comes after physics," as indicating a dark realm of the yet unknown, or even of the unknowable, which surrounds the clear zone of positive knowledge, into which we may peer, but will discern nothing. The objects of metaphysics-the soul, the cosmos, the deity - -are in this view vain imaginings, not objects of which there can be knowledge in the scientific meaning - that is, objects amenable to the experimental method. Such a view simply ignores the scientific tradition. Modern science is the result of the formulation and adoption of the experimental method, but the experimental method is not self-evident or inherently rational; it depends on a metaphysical concept, and its rationality can be established only by metaphysical principles. To contrast, then, the experimental method with the principles on which it depends, to describe one as the realm of science and the other as the realm of ignorance or unknowability, is from any philosophic point of view stultifying, and, in the literal sense, absurd.

What has made it possible to consider metaphysics as an unreal science, or as a realm of unreal fancy, is the peculiar position in regard to the natural sciences in which the purely mathematical sciences stand. Mathematics does not use the experimental method, and in the hierarchy of the sciences mathematics scems sufficient of itself for the foundation and support of the whole superstructure. But mathematics is only an abstract science of quantity; its concepts lack the one essential character which experimental science calls for-concreteness-and this metaphysics alone can supply.

The modern era of philosophy from Descartes onwards has been dominated by the insistence of the scientific problem-that is, the problem of No. 2677 , VOI. IO6] the ultimate nature of the reality we study in physical science by the experimental method. This interest in the nature of scientific reality replaces the main interest of the philosophy of the mediæval period, which was concerned with the origin and destiny of the human soul, and, more generally, with the relation of man to God. If modern philosophy may be said to join hands with the ancient philosophy of Greece, it is not in the identity of its interest; for, though the Greeks were mathematicians, they had no conception of the experimental method as we practise it, and it is even doubtful if it could have been made to appeal to them on the ground of rationality.

The principle of relativity is the direct outcome of the application of the experimental method, and the full force of its appeal is based on our absolute confidence in the metaphysical concept of reality which is the ground and reason of that method. The experimental method has taken possession of the modern mind, and it assumes for us something like the unmodifiable character of an instinct. If experiment proves a certain velocity to be constant under conditions which require us to predict its variation; if experiment shows the movement of a source of light to be without the expected effect on the velocity of propagation-well, it is our concept of the nature of reality which must adapt itself to the experiment. The prediction is based on the concept that space and time provide an absolute system of reference; the null result of the experiment negatives that concept, and henceforth space and time are "shadows"; they must vary, because under varying conditions velocity is constant.

Those who affirm that the principle of relativity is purely mathematical, and not metaphysical, and, therefore, resent the intrusion of metaphysics into the discussion of its equations, conceive the principle to be purely methodological, to be concerned only with abstract quantitative measurement, and merely to substitute a very complex and difficult set of equationformulæ for a discarded simpler one, in the interest of greater precision and accuracy alone. Those who take this view seem to me to misapprehend the significance of the principle. It is to be understood only when taken in its historical connection with the metaphysical constructions of the great philosophers.

Since Descartes, the speculations of philosophy have centred round the concepts of substance and 